\title{
Ensinando configurações do smartphone e aplicações sociais para o público 60+ por meio de aulas semanais e intervenções remotas
}

\author{
Kamila Rios da Hora Rodrigues, Isabela Zaine, \\ Brunella Della Maggiore Orlandi, Maria da Graça Campos Pimentel
}

\author{
${ }^{1}$ Instituto de Ciências Matemáticas e de Computação - Universidade de São Paulo (USP) \\ Avenida Trabalhador São-carlense, 400 - Centro - São Carlos - SP - Brasil \\ \{kamila.rios,mgp, \}@icmc.usp.br, brunella@alumni.usp.br, isabela.zaine@gmail.com
}

\begin{abstract}
Resumo. Este trabalho investiga os fatores que permitem ao idoso utilizar recursos tecnológicos para melhorar a sua qualidade de vida e envelhecimento, bem como apresenta os desafios associados a esse processo. O objetivo é escrever os princípios e a metodologia de um programa educacional digital de educação para idosos desenvolvido em colaboração interdisciplinar e suportado por um sistema e um aplicativo móvel para monitorar remotamente e promover a generalização da aprendizagem. Antes da participação no curso foi identificada uma frequência de uso do smartphone em suas funções básicas, porém, baixo senso de autoeficácia ou competência em usá-lo. O aplicativo móvel, chamado de "ajudante digital", foi utilizado como recurso didático e considerado um recurso válido por $76 \%$ dos participantes, uma vez que ele estimulou ou aumentou o envolvimento dos alunos com tarefas práticas enviadas para casa de modo remoto. Ao fim do estudo foi possível observar o interesse da população idosa presente nas aulas de letramento em utilizar dispositivos móveis e os aplicativos de comunicação e lazer oferecidos por ele. O uso de uma metodologia inovadora, que agrega um ajudante digital, facilitou o ensino e o aprendizado dos alunos, sobretudo no aprendizado de redes sociais (Facebook e WhatsApp). As aulas demonstraram a relevância de programas de alfabetização digital para idosos. Estudos futuros serão conduzidos para estabelecer comparações entre métodos específicos - como o aqui descrito, e métodos tradicionais para o letramento digital de idosos.
\end{abstract}

\section{Introdução}

A rápida evolução das tecnologias digitais leva a uma lacuna entre o potencial tecnológico e as competências e habilidades digitais das gerações anteriores. É necessário, portanto, que as tecnologias se tornem mais intuitivas para os idosos, de modo a dar continuidade e melhoria nos processos de aprendizagem humana na velhice [Hill et al. 2015, Peña-López et al. 2015].

Reconhece-se que a promoção da inclusão tecnológica e da alfabetização digital de idosos reduz as diferenças de idade no uso de tecnologias, mas, sobretudo, impacta outras fontes de desigualdades sociais decorrentes de níveis de educação e renda, diferenças de gênero e acesso à cultura e lazer [Huber and Watson 2014, Neves et al. 2018]. Para as gerações mais jovens, o desenvolvimento de competências digitais já faz parte dos currículos da educação formal. A promoção tardia da inclusão de idosos na era digital requer mais do que facilitar o acesso, mas também a melhoria dos processos de aprendizagem que, para esse grupo, ocorrem preferencialmente em contextos educativos não formais.

No Brasil, a Universidade Aberta ao Idoso (UATI) representa espaços socialmente reconhecidos de aprendizagem para idosos e onde se operacionaliza o conceito de "Aprendizagem ao Longo da Vida" [Peña-López et al. 2015]. Nesses espaços oportunidades educacionais para o desenvolvimento de competências tecnológicas e digitais têm se tornado frequentes diante da grande demanda de idosos por atividades dessa natureza.

Pressupõe-se que os programas de alfabetização digital para idosos em ambientes universitários atuem como meio de potencializar o uso das tecnologias de comunicação e informação móveis, também com a expectativa de minimizar os efeitos negativos relacionados ao seu uso, como insegurança e risco 
à privacidade e proteção pessoal [del Pilar Díaz-López et al. 2016, ten Bruggencate et al. 2019]. A literacia digital (ou letramento digital) desempenha um papel significativo na vida pessoal e coletiva dos idosos, na partilha de informações e novos conhecimentos, que se traduz na melhoria do bem-estar e qualidade de vida, na redução dos níveis de depressão e ansiedade, e na redução do impacto negativo do isolamento e solidão entre os usuários mais velhos, sobretudo no momento atual de pandemia do COVID-19. Por outro lado, a falta de acesso, o uso indevido ou o abuso de tecnologia pode ser prejudicial e dificultar interações competentes e seguras [Peña-López et al. 2015].

As metodologias e a implementação de ações em alfabetização digital de idosos ainda não estão bem documentadas na literatura de pesquisa [Kachar 2010, Chiu et al. 2019]. Na perspectiva da Gerontologia e da Computação, entende-se que as estratégias de letramento digital para idosos devem considerar as mudanças associadas ao envelhecimento biopsicossocial, seus interesses, motivações e objetivos, bem como as características dos dispositivos tecnológicos e sua usabilidade. Os métodos devem contemplar a ampliação da capacidade de generalização da aprendizagem e sua aplicação em diversos contextos favorecendo também o senso crítico dos idosos no uso das tecnologias [Raymundo and Castro 2020].

Considerando a oferta de atividades de letramento digital neste contexto, o objetivo deste artigo é descrever uma metodologia de intervenção planejada em colaboração multidisciplinar. Serão apresentados os princípios e a organização didática da intervenção, destacando o diferencial do ponto de vista adotado e os principais aplicativos utilizados.

O artigo está dividido da seguinte maneira: a Seção 2 discorre sobre o letramento digital para idosos, o sistema ESPIM e o ajudante digital que o compõe, a Seção 3 descreve os procedimentos do programa de ensino, Seção 4 contém os resultados do emprego da metodologia e a Seção 5 descreve as considerações finais.

\section{Letramento Digital para Idosos}

O programa de intervenção educacional aqui descrito visa oferecer oportunidades de letramento digital na utilização de dispositivos móveis (smartphones e tablets) para idosos envolvidos na educação não formal. Esse programa está também associado ao desenvolvimento de um projeto de investigação mais amplo, estabelecido por uma parceria multidisciplinar entre profissionais e investigadores das áreas de Gerontologia e da Computação da Universidade de São Paulo (USP). A parceria estabelecida permitiu o desenvolvimento de uma metodologia partilhada desde a estrutura das aulas, conteúdos, tempo de execução e a inclusão de um recurso didático e experimental composto por um aplicativo móvel chamado de "ajudante digital". Esse ajudante é suportado por um método chamado ESPIM (Experience Sampling and Programmed Intervention Method) [Cunha et al. 2021]. O ESPIM conta com o apoio de um infraestrutura computacional de mesmo nome que é composta por uma interface web de autoria e um aplicativo móvel [Cunha et al. 2021, Rodrigues et al. 2018].

O programa de intervenção era oferecido por meio das ações de extensão universitária e foi submetido à aprovação do Comitê de Ética em Pesquisa com Seres Humanos da USP.Como grande uma parte significativa da metodologia programa é suportada pelo ESPIM, esse recurso será descrito a seguir.

\subsection{Sistema ESPIM}

O ESPIM - Experience Sampling and Programmed Intervention Method - é apresentado como um método apoiado em uma solução de software que oferece alternativas para coleta de dados e intervenções programadas por especialistas de diferentes áreas, tais como Saúde e Educação [Cunha et al. 2021, Rodrigues et al. 2018]. Consiste, portanto, de uma aplicação Web de autoria e um aplicativo móvel. O sistema permite o planejamento e a autoria de intervenções com base em dados coletados de forma explícita, bem como a troca de dados entre as diferentes partes envolvidas, como professores e alunos [Cunha et al. 2021, Rodrigues et al. 2018]. 
A concepção do ESPIM foi inspirada em procedimentos da Psicologia e da Computação Ubíqua. Uma das influências dentro da área da Psicologia foi o Experience Sampling Method - ESM, que opera formas de coleta de informações sistêmicas sobre a experiência das pessoas no ambiente natural [Csikszentmihalyi and Larson 2014]. Há momentos, no entanto, em que, além da coleta de dados, existe o interesse de intervir de alguma forma na situação, como forma de provocar alguma mudança de comportamento. Assim, uma segunda influência na concepção do ESPIM vem da área de Análise do Comportamento [Willems 1974], mais especificamente da área de Programação de Ensino. Os princípios, procedimentos e tecnologias produzidos por essa área são generalizáveis para diferentes contextos, cuja finalidade é instalar ou alterar comportamentos, ou diminuir a ocorrência dos mesmos.

A interface web do ESPIM permite a criação de planos individualizados de intervenções programadas, cadastro de participantes e visualização dos resultados enviados ao servidor. Por meio dela o pesquisador pode programar diferentes formas de interação entre os participantes e o sistema, tanto no recebimento quanto no envio de informações (entre elas mídias). Programas de intervenção podem ser criados consistindo em questões abertas, solicitação de mídias, múltipla escolha, escalas Likert e mensagens instrucionais/informativas. O fluxo entre uma pergunta e outra pode ser diferente de acordo com a resposta fornecida pelo usuário. As perguntas podem ser programadas nos formatos de texto, áudio ou vídeo e os participantes podem respondê-las em qualquer um desses formatos. AS respostas são disponibilizadas ao pesquisador automaticamente após serem produzidas [Cunha et al. 2021].

O pesquisador também define, através da interface $\mathrm{Web}$, o intervalo em que o programa estará ativo para realizar intervenções ou recolha de dados. O sistema permite que os participantes recebam notificações sonoras, que podem variar em frequência e intensidade, caso haja uma solicitação agendada no sistema. Para tanto, é configurado um alarme para ser acionado em um horário específico, determinado pelo pesquisador. $\mathrm{O}$ alarme serve como um lembrete para os participantes interagirem com o aplicativo [Rodrigues et al. 2018]. O aplicativo móvel gera telas, de acordo com as informações programadas no sistema Web, para que o participante possa interagir em seu dispositivo móvel [Cunha et al. 2021].

No contexto de intervenções educativas em letramento digital para idosos - na tentativa de preencher o hiato digital nessa faixa etária e fortalecer as redes sociais, a autonomia, o pertencimento, o bem-estar e a qualidade de vida - a utilização do ESPIM amplia o caráter metodológico e possibilidades didáticas com ênfase no aprendizado da tecnologia pela própria tecnologia (neste caso, via smartphones) em um ambiente natural. Tem ainda o potencial para estimular a generalização da aprendizagem e a sua aplicação a contextos extraclasse, além de promover o acompanhamento remoto dessa aprendizagem por tutores ou professores.

\section{Procedimentos do Programa de Ensino}

\subsection{Organização}

Para o curso de letramento as salas de aula foram instaladas nas próprias unidades de ensino superior. O programa foi elaborado para ocorrer durante um semestre. Os encontros aconteciam uma vez por semana, com duas horas de aula, totalizando 15 aulas, 15 semanas. O conteúdo programático previa aulas de configuração do próprio smartphone em que se ensinava: Fazer e receber chamadas; Adicionar, editar e excluir contatos; Lista telefônica; Fotografar, filmar; Configurações como ícones, brilho da tela, tamanho da fonte, tempo ocioso, senha de segurança, chamada de emergência; Excluir fotos e vídeos. Além disso, os aplicativos Play Store, Google Search, Facebook e WhatsApp eram ensinados. De modo específico, para o Facebook era ministradas aulas sobre: Criar uma conta; Editar perfil - foto e capa; Adicionar amigos; Publicações - públicas e privadas; Adicionar imagens e/ou fotos; Adicionar local e check-in; Comentar, curtir e compartilhar; Messenger - mensagem privada, chamada de áudio e vídeo. Para o WhatasApp eram ministradas aulas sobre: Fazer e receber chamadas de voz e vídeo; Áudio e mensagem de texto; Adicionar contatos; Grupos; Anexar mídia e fotos; Compartilhar localização, contato; Responder, encaminhar e excluir mensagens específicas. 
As aulas eram compostas pelos alunos idosos matriculados no curso que faziam parte do critérios de inclusão do projeto (ser 60+ e ter um smartphone Android), e pelos monitores e coordenadores (estudantes universitários) que variaram em número conforme o semestre. Assim, todas as aulas contaram com a participação de pelo menos um líder do curso, bem como o grupo de monitores responsáveis pelas aulas. Os monitores, conforme referido anteriormente, eram estudantes universitários, licenciados em Gerontologia ou Computação, e os coordenadores, alunos de doutorado ou pós-doutorado, envolvidos no projeto.

\subsection{Preparação da aula}

Todas as aulas foram pensadas e organizadas de forma colaborativa com todos os membros da equipe, em reuniões semanais ao longo do processo. Após cada conteúdo abordado, eles foram elaborados em formato expositivo, incluindo atividades práticas. A apresentação do conteúdo, em power point, foi montada com imagens exportadas das telas dos smartphones para facilitar a localização dos ícones nos dispositivos móveis pelos idosos. O material desenvolvido foi impresso e organizado em apostilas que eram entregues aos alunos no início de cada aula. Desta forma, todos receberam material de apoio tanto para anotações pertinentes dentro da sala de aula quanto para servir de complemento ao estudo fora da sala de aula.

As atividades práticas foram realizadas individualmente ou em grupo, dependendo do tema da aula. Foram adotadas atividades individuais, por exemplo, na inclusão, exclusão e edição de contatos por se tratarem de recursos pessoais. Nesses momentos, os monitores atuavam como auxiliares, esclarecendo dúvidas e orientando cada aluno quando sentia dificuldades. Essa ajuda sempre ocorreu para orientá-los e nunca para realizar as tarefas por eles. Para as atividades coletivas, a seleção dos componentes do grupo foi feita previamente com o intuito de garantir um equilíbrio na questão do conhecimento e, com isso, promover a troca de experiências, solidariedade e companheirismo. Para essa estrutura de grupo, cada monitor fazia parte de uma equipe. Para atividades tais como baixar e experimentar aplicativos de jogos, bem como falar sobre a confiabilidade dos aplicativos, foi usado as dinâmicas de grupo.

Para módulos de redes social, como WhatsApp e Facebook, foram desenvolvidas estratégias diferenciadas. Para o WhatsApp, a partir da segunda aula sobre o tema, foram criados grupos para cada turma. Esses grupos foram formados a fim de serem utilizados como meio para algumas intervenções remotas solicitadas pelo ESPIM e também como meio de divulgação de notícias e retirada de dúvidas. Além disso, os grupos no WhatsApp proporcionaram a aproximação e o compartilhamento de importantes informações entre os próprios idosos e ainda hoje os mesmos são ativos. Em relação ao Facebook, de acordo com o mesmo propósito de realizar as intervenções remotas, foram construídos dois perfis fictícios, o primeiro chamado 'Onlainina' e, devido à dificuldade dos idosos com a palavra 'onlainina', o segundo perfil chamado 'Quarentine', usado nos semestres em que as aulas aconteceram de maneira remota devido ao distanciamento social provocado pelo COVID-19. Esses perfis enviaram mensagens, comentaram e/ou reagiram às postagens dos alunos, dando exemplos e compartilhando informações que ajudaram no trabalho de treinamento e interatividade.

\subsection{Intervenções Remotas}

As intervenções à distância tiveram o papel de tarefas complementares e permitiram o exercício prático dos conteúdos e competências apresentados e treinados em sala de aula. Cinco tarefas eram enviadas durante a semana, via "ajudante digital", uma para cada dia. Dentre as cinco intervenções remotas, pelo menos uma representava algum conteúdo relacionado às aulas anteriores que foram chamadas de intervenção de manutenção, e recapitulavam tal conteúdo. As cinco intervenções remotas foram disparadas de forma interativa, de modo que os alunos do curso pudessem realizar suas tarefas fora da sala de aula, em momento de sua preferência e com autonomia. Os alunos foram treinados quando ao uso e funcionalidades do "ajudante". 
Todas as intervenções remotas foram enviadas pelo aplicativo ajudante.O aplicativo móvel emitia um som intermitente por 30 segundos às 19 horas diariamente (horário determinado com os alunos), como um lembrete para o aluno realizar as tarefas do dia. Os idosos podiam fazer todas de uma vez, aos poucos, como uma tarefa por dia, e repeti-las sempre que quisessem. Configurações de acessibilidade do celular foram realizadas nos dispositivos do idoso para ampliar as fontes usadas nos programas de intervenção via "ajudante digital".

\subsection{Verificação de intervenções remotas}

A verificação das atividades realizadas remotamente era considerada uma etapa importante em todo o processo de ensino. No início de cada aula, entre 15 e 30 minutos era dedicado para que os monitores verificassem junto aos idosos se a atividade havia sido recebida, se o aplicativo tocou para lembrá-los de realizar as tarefas, se eles precisaram de ajuda para realizá-las e que tipo de ajuda foi essa (familiares, amigos, o material impresso) e se ainda tinham dúvidas sobre o conteúdo. A conferência era feita individualmente, e o aluno era solicitado à mostrar, em seus próprios dispositivos, as respostas realizadas nas intervenções remotas recebidas. Quando o aluno não conseguia ou não completava a tarefa, o monitor revisava passo a passo com ele para reforçar e esclarecer quaisquer questões pendentes. A frequência e a quantidade das atividades também foram anotadas na planilha especificando o número de vezes de execução, uma vez que todos foram incentivados a praticar as atividades recebidas mais de uma vez.

\subsection{Aulas de revisão}

$\mathrm{Na}$ metade das aulas do curso e ao final dele foram ministradas aulas de revisão do conteúdo. Entre os conteúdo com maior índice de dúvidas, de aprendizado parcial ou não aprendizado, estão aqueles relacionados às redes sociais.

Destaca-se aqui os conteúdos com maior índice de dúvidas para as aulas de revisão: a) Facebook (alterar foto do perfil, enviar mensagem privada $x$ enviar mensagem pública, realizar publicações com fotos e localização ou marcando amigos e, realizar chamadas de áudio/vídeo); b) WhatsApp (responder uma mensagem para alguém em específico - em um grupo, fazer chamadas de vídeos, gravar mensagem de áudio, achar uma foto na galeria para enviar, compartilhar a localização, excluir mensagens).

Os idosos inicialmente tinha dúvidas no entendimento dos ícones e das funcionalidades, embora esses representem funções parecidas com as do cotidiano. Esses idosos enfrentavam dificuldades não só no entendimento, mas também na localização dos itens e na segurança em usá-los. Por não serem nativos digitais, levam um tempo para o entendimento do novo paradigma de interação. A experiência dos coordenadores deste curso ao longo de 10 semestres de oferta do mesmo, aponta, no entanto, que nas última aulas os idosos costumam estar mais confiantes e se arriscam a errar, pois perderam o receio de causar algum erro irreparável. Também aprendem a entender a representação de cada ícone na tela.

Nos três últimos semestres o curso tem sido realizado de modo online e um dificultador foi inserido, a ferramenta para ministrar aulas remotas. Notou-se, entretanto, que devido ao aprendizado acelerado causado pelo uso de tecnologias de comunicação durante a pandemia, os idosos estão chegando ao curso com maior facilidade de usar algumas das funcionalidades supracitadas, entre elas: fazer chamadas em vídeos, encontrar imagens na galeria para envio ou postagem e gravar áudios. Como alguns dos ícones são semelhantes entre os aplicativos, notou-se que a partir da segunda aula os idosos já não têm mais dificuldades para usar o Google Meet e conseguem fazer a chamada, bem como desligar e ligar sua imagem e som. Foi uma aceleração de aprendizado imposto pela pandemia que permitiu certo avanço na inclusão digital do público idoso. Sabe-se, no entanto, que esse cenário não é o padrão em todas as fronteiras do Brasil.

\section{Resultados do emprego da metodologia}

Entre 2017 e 2021 participaram do curso presencial em torno de 250 alunos idosos, com 60 anos ou mais. Eram em sua maioria do sexo feminino $(64,7 \%)$ com idade média de 68,60 (+ 5,82 DP) anos. Pre- 
valência de casados ou em união estável (43,7\%), seguidos de viúvos(as) (23,5\%), solteiros(as) (17,5\%) e divorciados(as) ou separados(as) (15,3\%). Da amostra total, 35\% dos idosos moravam sozinhos. O grau de escolaridade foi caracterizado em anos de estudo formal, ou seja, anos de aprendizagem realizada em instituições de ensino indicando 10,77 + 5,07 (M + DP) anos de estudo formal. Quanto à renda familiar, $61,8 \%$ dos entrevistados indicaram renda de até quatro salários mínimos. Em relação à aposentadoria, já estava aposentados 120 (72,3\%), e 74\% dos entrevistados não exerciam atividades profissionais remuneradas. A maioria dos idosos possuía wi-fi $(80,3 \%)$ e/ou rede móvel de dados $(82,6 \%)$. A frequência de uso de dispositivos móveis revelou que uma pequena proporção dos alunos nunca usou dispositivos móveis. Os alunos que utilizaram dispositivos móveis com frequência ou sempre, representaram $87,3 \%$, porém $55,2 \%$ relataram ter pouca experiência neste uso. Muitos $(84,1 \%)$ solicitaram ajuda de outras pessoas para atingir seus objetivos ao usar seu dispositivo.

Quanto ao uso do ajudante digital, 72,9\% dos participantes encontraram vantagens no uso do aplicativo. Da mesma forma, 70,6\% apresentaram maior engajamento nas atividades e no uso do dispositivo móvel. A assiduidade às aulas foi de $82,2 \%$ com assiduidade em 10 ou mais aulas. Dificuldades no uso do aplicativo foram relatadas por $24,8 \%$ dos alunos e $35 \%$ solicitaram ajuda de familiares ou amigos para utilizá-lo. Em relação à forma de alerta sonoro do aplicativo, 67,8\% acharam útil, 9\% disseram que era indiferente e $4 \%$ se incomodaram com o alerta sonoro do aplicativo.

\section{Considerações Finais}

Este estudo foi motivado pela necessidade de construção de um programa de alfabetização digital para a população idosa com base nos preceitos da ALV (Aprendizado ao Longo da Vida) e de acordo com as recomendações encontradas na UNESCO, uma vez que os avanços tecnológicos exigem novas competências e é necessário seguir essas novas demandas ao longo da vida.

A importância deste estudo, na área do letramento digital, é reiterada por sua inovação e novidade, por utilizar uma aplicação no aprendizado da própria tecnologia, desenvolvida de forma interdisciplinar por profissionais de Computação e Gerontologia envolvidos em programas universitários. É importante ressaltar a importância de um programa educativo específico voltado para a alfabetização digital de idosos com suas peculiaridades, subsídios teóricos e práticos para uma reflexão contínua sobre o assunto.

[Chiu et al. 2019] mencionam que embora muitos estudos relevantes demonstrem a importância da inclusão na era digital para os idosos, poucos se propõem a discutir a metodologia dos programas de ensino-aprendizagem.

As demandas para idosos têm sido utilizadas de forma geral e frequente em diversos segmentos (saúde, locomoção, consultas, cuidados, etc.) inclusive em pesquisas científicas sinalizando bons resultados. [Neves et al. 2018] em estudo realizado em Portugal e Toronto, utilizaram uma aplicação de combate à solidão e isolamento de idosos institucionalizados ou em risco, facilitando a comunicação entre familiares e amigos, e obtiveram resultados favoráveis. [Zaine et al. 2019], de modo parecido, usou o aplicativo do ESPIM para promover a aproximação e conexão social entre idosos e seus amigos e familiares em estudos conduzidos no Brasil e Reino Unido.

Neste curso, a estimulação e o monitoramento por meio do sistema ESPIM e seu aplicativo na utilização de dispositivos móveis (smartphones), mostrou-se um mecanismo inovador de práticas pedagógicas em letramento digital voltado para idosos. A utilização do aplicativo, conforme demonstrado nos resultados, incentivou e promoveu um maior engajamento no uso de smartphones, potencializando um maior contato com as tarefas, o que pode tornar o processo de recapitulação e revisão de conteúdo eficiente e o treinamento eficaz. Entende-se, portanto, que essa metodologia despertou interesse e motivação para uso fora da sala de aula. A interatividade parece ter sido um fator relevante no aumento da frequência de uso de dispositivos móveis, o que enfatiza o interesse por novas estratégias de aprendizagem.

Em pesquisa recente, [ten Bruggencate et al. 2019] entendeu que intervenções direcionadas à 
população idosa, com o objetivo de inseri-los digitalmente, não são satisfatórias. O fato de o envelhecimento ser heterogêneo, com necessidades sociais e pessoais próprias de cada indivíduo, não é levado em consideração na execução desses programas. [Narushima et al. 2018] e [Hill et al. 2015] ponderaram sobre os efeitos negativos relatados pelos idosos, como insegurança, ansiedade e insatisfação quando o curso oferecido não é adequado ou quando os profissionais não estão preparados para atender à essa população. No entanto, os mesmos autores mencionam benefícios ligados à aprendizagem ao longo da vida como aumento do engajamento social, melhoria do bem-estar, aumento do suporte social e estimulação das funções cognitivas quando essas são projetadas para atender às expectativas dos idosos. De acordo com os autores, a existência de um curso para o processo de inclusão digital e social do idoso não pode ser única; deduz-se que cada indivíduo tem suas exigências, necessidades e expectativas, alunos ou professores. Porém, os profissionais que atuam na área precisam entender como e quem são seus alunos antes de iniciar qualquer intervenção. Nesse caso, o programa também discutido no estudo aqui apresentado, é um curso básico de alfabetização e, portanto, um passo à frente das escolhas críticas e pessoais; traz informações e procedimentos básicos de manuseio, gestão, segurança e a possibilidade de oferecer suporte para iniciar o processo de utilização de dispositivos móveis e aprimorar as habilidades e competências necessárias para preparar o idoso para fazer suas próprias escolhas de forma segura e consciente.

É fundamental enfatizar que o simples fato de possuir ou utilizar o dispositivo móvel não confirma o efetivo letramento digital. Fato atestado pelos próprios idosos ao responderem sobre a experiência no uso de dispositivos móveis: $74,1 \%$ dos pesquisados relataram ter pouca ou nenhuma experiência de uso, mesmo tendo acesso (84,8\% indicaram usar o dispositivo móvel sempre ou com frequência). Ser incluído é conectar as redes da Internet com interesse e finalidade; participar, usar como forma de trabalhar, poder se relacionar de forma segura e consciente com um mundo novo e sem limites que é o mundo da tecnologia [Neves et al. 2018, Raymundo and Castro 2020].

Portanto, programas educacionais que abordem questões básicas como terminologia, manuseio, configuração de dispositivos móveis passam a ser tão importantes quanto trabalhar o significado do uso das tecnologias no contexto de vida do idoso aliado ao seu uso de forma inclusiva e saudável [Padilla-Góngora et al. 2017, Neves et al. 2018]. Para isso, deve-se levar em consideração a velocidade de aprendizagem, a necessidade de constantes recapitulações e revisões, além da motivação, propósito e satisfação com a vida [Kachar 2010, Jin et al. 2019].

Novas aptidões e competências são necessárias aos idosos - imigrantes digitais - para a aquisição de uma nova linguagem, desde mecanismos de reconhecimento e codificação até seu uso crítico garantindo a participação na sociedade [Borges 2017, Peña-López et al. 2015].

Limitações deste trabalho incluem o estudo de idosos em aulas presenciais e da região Sudeste. As aulas remotas atuais, no entanto, estão sendo assistidas por alunos de todas as partes do Brasil e, como trabalhos futuros, pretende-se investigar semelhanças e diferenças entre os perfis de idosos participantes dos cursos de letramento da USP no programa da UATI.

\section{Agradecimentos}

À Fundação de Amparo à Pesquisa do Estado de São Paulo - FAPESP pelo financiamento de parte deste Projeto - Proc. n²017 / 19915-0. Aos idosos que participaram do programa de letramento digital e a todos os alunos e profissionais que contribuíram com este estudo.

\section{Referências}

Borges, F. G. B. (2017). A construção de uma metodologia para o letramento digital. Raído, 11(25):280-294.

Chiu, C.-J., Tasi, W.-C., Yang, W.-L., and Guo, J.-L. (2019). How to help older adults learn new technology? results from a multiple case research interviewing the internet technology instructors at the senior learning center. Computers \& Education, 129:61-70. 
Csikszentmihalyi, M. and Larson, R. (2014). Validity and reliability of the experience-sampling method. In Flow and the foundations of positive psychology, pages 35-54. Springer.

Cunha, B. C. R., Rodrigues, K. R. D. H., Zaine, I., da Silva, E. A. N., Viel, C. C., and Pimentel, M. D. G. C. (2021). Experience sampling and programmed intervention method and system for planning, authoring, and deploying mobile health interventions: Design and case reports. Journal of medical Internet research, 23(7): 24278 .

del Pilar Díaz-López, M., López-Liria, R., Aguilar-Parra, J. M., and Padilla-Góngora, D. (2016). Keys to active ageing: new communication technologies and lifelong learning. SpringerPlus, 5(1):1-8.

Hill, R., Betts, L. R., and Gardner, S. E. (2015). Older adults' experiences and perceptions of digital technology:(dis) empowerment, wellbeing, and inclusion. Computers in Human Behavior, 48:415423.

Huber, L. and Watson, C. (2014). Technology: Education and training needs of older adults. Educational Gerontology, 40(1):16-25.

Jin, B., Kim, J., and Baumgartner, L. M. (2019). Informal learning of older adults in using mobile devices: A review of the literature. Adult Education Quarterly, 69(2):120-141.

Kachar, V. (2010). Envelhecimento e perspectivas de inclusão digital. Revista Kairós: Gerontologia, 13(2).

Narushima, M., Liu, J., and Diestelkamp, N. (2018). I learn, therefore i am: A phenomenological analysis of meanings of lifelong learning for vulnerable older adults. The gerontologist.

Neves, B. B., Waycott, J., and Malta, S. (2018). Old and afraid of new communication technologies? reconceptualising and contesting the 'age-based digital divide'. Journal of Sociology, 54(2):236248.

Padilla-Góngora, D., López-Liria, R., del Pilar Díaz-López, M., Aguilar-Parra, J. M., Vargas-Muñoz, M. E., and Rocamora-Pérez, P. (2017). Habits of the elderly regarding access to the new information and communication technologies. Procedia-Social and Behavioral Sciences, 237:1412-1417.

Peña-López, I. et al. (2015). Rethinking education. towards a global common good?

Raymundo, T. M. and Castro, C. d. S. S. (2020). Analysis of a training program for older workers in the use of technology: difficulties and satisfaction. Revista Brasileira de Geriatria e Gerontologia, 22.

Rodrigues, K. R., Cunha, B. C., Zaine, I., Viel, C. C., Scalco, L. F., and Pimentel, M. G. (2018). Espim system: interface evolution to enable authoring and interaction with multimedia intervention programs. In Proceedings of the 24th Brazilian Symposium on Multimedia and the Web, pages $125-132$.

ten Bruggencate, T., Luijkx, K., and Sturm, J. (2019). How to fulfil social needs of older people: Exploring design opportunities for technological interventions. Gerontechnology, 18(3):156-167.

Willems, E. P. (1974). Behavioral technology and behavioral ecology. Journal of Applied Behavior Analysis, 7(1):151-165.

Zaine, I., Frohlich, D. M., Rodrigues, K. R. D. H., Cunha, B. C. R., Orlando, A. F., Scalco, L. F., and Pimentel, M. D. G. C. (2019). Promoting social connection and deepening relations among older adults: design and qualitative evaluation of media parcels. Journal of medical Internet research, 21(10):e14112. 